\title{
Cis-regulatory programs in the development and evolution of vertebrate paired appendages
}

\author{
Andrew R. Gehrke ${ }^{a}$ and Neil H. Shubin ${ }^{\mathrm{a}^{*}}$ \\ ${ }^{a}$ Department of Organismal Biology and Anatomy, The University of Chicago, Chicago, IL 60637, USA \\ agehrke@uchicago.edu, *nshubin@uchicago.edu (corresponding author)
}

\begin{abstract}
Differential gene expression is the core of development, mediating the genetic changes necessary for determining cell identity. The regulation of gene activity by cisacting elements (e.g., enhancers) is a crucial mechanism for determining differential gene activity by precise control of gene expression in embryonic space and time. Modifications to regulatory regions can have profound impacts on phenotype, and therefore developmental and evolutionary biologists have increasingly focused on elucidating the transcriptional control of genes that build and pattern body plans. Here, we trace the evolutionary history of transcriptional control of three loci key to vertebrate appendage development $(F g f 8, S h h$, and $H o x D / A)$. Within and across these regulatory modules, we find both complex and flexible regulation in contrast with more fixed enhancers that appear unchanged over vast timescales of vertebrate evolution. The transcriptional control of vertebrate appendage development was likely already incredibly complex in the common ancestor of fish, implying that subtle changes to regulatory networks were more likely responsible for alterations in phenotype rather than the de novo addition of whole regulatory domains. Finally, we discuss the dangers of relying on inter-species transgenesis when testing enhancer function, and call for more controlled regulatory swap experiments when inferring the evolutionary history of enhancer elements.
\end{abstract}

Keywords: gene regulation, evo devo, fin-to-limb, transgenesis, developmental systems drift, topological domain

\section{Contents}

1. Introduction

2. Fgf8 enhancers in phylogeny: conservation, flexibility, and redundancy

3. Shh regulation in appendages: conservation of landscape and function

4. The regulatory landscape of Hox genes in appendage evolution

5. Inter-species transgenesis and developmental systems drift

6. Conclusions

7. Future directions: a phylogenetic perspective on functional genomics Acknowledgements References 


\section{Introduction}

The vast majority (> 95\%) of mammalian genomes consist of sequence that does not code for proteins, and much of this non-genic sequence harbors regulatory elements that control the activity of surrounding target genes. Genomic regions that drive specific patterns of target gene expression in time and space are deemed "enhancers" or "CisRegulatory Elements" (CREs), and consist of a series of short ( 10 bp) transcription factor (TF) binding sites that can vary in both length and number for any given target gene [1-3]. As vertebrate genes involved in development are largely conserved (e.g., Carroll, 2008), there has been increasing interest in CREs as the potential substrate for the evolution of morphological features [4]. Evolutionary developmental biology ("evodevo") has seen an increasing number of examples revealing how even slight changes to a CRE can have profound effects on phenotypes [5-7].

Recent advances in functional genomics have allowed biologists to move beyond a snapshot of activity for a single CRE to identification and functional analyses of entire suites of CREs. Chromosome conformation capture techniques (e.g. 4C, 5C, HiC) provide the interaction profiles of specific loci or even entire genomes, without prior knowledge of enhancer-promoter interaction [8]. ChIP-seq can identify genome-wide binding profiles for a specific protein or provide patterns of histone modifications, and ATAC-seq can rapidly identify specific areas of open chromatin at the level of transcription factor binding sites $[9,10]$. Thus, a more holistic picture of gene regulation is beginning to arise, revealing a dynamic and complex landscape of enhancer activity spread over hundreds of thousands or even millions of base pairs amidst changing landscapes of chromatin in different tissues types and species [11]. To complement the array of functional genomic techniques, we are experiencing an influx of whole genome sequence from across the tree of life. Thus, evolutionary and developmental biologists are beginning to identify, characterize, and manipulate entire complements of regulatory elements that are active in an embryonic structure of interest, and compare them among taxa in a phylogenetically-informed way. Armed with these data, we can ask: how are cis-regulatory programs orchestrated both between loci and across taxa?

The fins of bony fish and the limbs of tetrapods are composed of fundamentally distinct anatomies (Fig. 1B). How the pectoral fins of fish evolved into the limbs of tetrapods is a fundamental issue in evolutionary biology. Recent work in this field has increasingly turned attention to how modifications to regulatory regions have influenced the fin to limb transition. In this review, we summarize the regulatory architecture of three loci crucial to building the vertebrate appendage to understand how these programs have been assembled and deployed to build and pattern fins and limbs. These loci were chosen due to their established roles in shaping limb morphology (see mutants in Fig. $1 \mathrm{C})$, and their regulatory control has been studied at some level. For each gene ( $F g f 8$ and $S h h$ ) and locus (HoxD and HoxA gene clusters), we begin by summarizing its role in tetrapod limb development and what is known about its transcriptional control in mouse. We then compare these regulatory programs to those enacting upon the orthologous locus in a variety of fish species. We discuss how regulatory regions might have been assembled over time and the potential implications of changes to these regions for the fin to limb transition. Overall, comparisons of regulatory programs between fish and tetrapods reveal complex and dynamic regulatory landscapes that have been deeply 
conserved in throughout vertebrate evolution. While large domains of regulatory regions appear conserved, some loci appear to tolerate a high degree of enhancer position shuffling, while enhancers at other loci seem "locked" in their position. We end by discussing challenges in understanding the evolution of enhancer function across large phylogenetic distances, and call for increased attention to potential trans-regulatory changes when interpreting cross-species transgenics.

\section{2. $F g f 8$ enhancers in phylogeny: conservation, flexibility, and redundancy}

In tetrapods, limb outgrowth is driven by a distal structure of ectodermal cells called the "apical ectodermal ridge" (AER), the removal of which results in the elimination of distal structures in a stage-specific manner (Fig. 1). Studies in chick identified members of the Fibroblast Growth Factor $(F g f)$ family as being important for AER function, as adding beads soaked in $F g f$ proteins could rescue removal of the AER in chick limbs $[12,13]$. While multiple $F g f$ genes $(F g f 4, F g f 9, F g f 17)$ are active in the AER, $F g f 8$ is the only member expressed at the onset of the AER and throughout all cells of the structure [14]. In addition, $F g f 8$ seems to be the only member whose activity is essential for limb development (Fig. 1)[14].

As the AER (of which $F g f 8$ is a crucial component) is necessary for proper limb development, considerable attention has been taken to understanding how changes to AER dynamics may have influenced the evolution of vertebrate appendages $[15,16]$. An AER with active $f g f 8$ has been revealed in a variety of fish species, where it shares a similar role with its murine ortholog to drive appendage bud outgrowth [17-19]. One noticeable difference when compared to tetrapod limbs is the transition from an AER to an apical fold (AF) in fish, where the AER transforms into a layer of cylindrical cells that will eventually build the actinotrichia (supporting structures) and lepidotrichia (rays) of fins [19]. This transition is important because it marks the addition of dermal skeleton to the endochondral elements of the proximal fin (Fig. 1). As the tetrapod limb consists only of endochondral bone, one potential hypothesis for the fin to limb transition is the postponing (or elimination) of the AF by extension of AER activity, possibly mediated by prolonged or bolstered $f g f 8$ [15]. Thus, understanding how $f g f 8$ is regulated in appendages across vertebrates may shed light on how changes in gene expression in the AER have influenced the fin to limb transition.

In mouse limbs, $F g f 8$ expression in the AER is controlled by a series of enhancers that lie centromeric to the gene, spanning a region of $\sim 170 \mathrm{~kb}$. Marinic et al. identified 5 enhancers (CE58, 59, 61, 66, 80) using phylogenetic footprinting that drove distinct patterns of AER expression in transgenic mice (Fig. 2) [20]. These are an addition to two previously described elements (CR3 and CR4) by Beerman and colleagues that also drove transgene AER expression [21]. These studies reveal that $F g f 8$ expression in the mouse limb is controlled by at least 7 distinct enhancers that drive nearly identical expression in a narrow strip of cells that make up the AER (Fig. 2). Moreover, the enhancers lie in a relatively gene-dense region, where the nearby "bystander" genes Poll, Dpcd, and Fbxw4 somehow escape the activity of these AER enhancers and are instead expressed at ubiquitous low levels [20]. These studies present a complex, layered regulation of $F g f 8$ in the AER of mouse limb buds. 
To understand how $f g f 8 a$ is regulated in developing pectoral fins, Komisarczuk et al. used phylogentic footprinting to identify a series of candidate enhancers in zebrafish [22]. The authors found 11 conserved sequences that, when introduced into as reporter constructs into zebrafish, drove expression in a distinct AER pattern (Fig. 2). Of these regions, 4 of their murine orthologs were tested in mouse in other studies (dr11/CE62, dr10/CE64, dr1/CR3, dr17/CE80), yet only two of these regions (dr17/CE80 and dr1/CR3) were active in both mouse limbs and fish fins [20-22]. The zebrafish elements dr11 and dr10 drove expression in fins, but their counterparts in mouse did not drive limb expression, which could be due to either a loss of activity of these elements in the tetrapod lineage, or simply local position effects of the reporter cassette in the transgenic animal [20, 22]. Komisarczuk and colleagues identified an additional 7 zebrafish CREs that drove AER expression that did not overlap with those tested in previous mouse studies [22]. The majority of these elements have orthologs in mouse, raising the potential number of shared $F g f 8$ enhancers between tetrapods and bony fish to 13 (Fig. 2).

Recently, genome sequences of a variety of non-model vertebrates have become available, allowing a more refined understanding of how the $F g f 8$ locus and its regulatory elements have evolved. Teleosts (bony fish, including zebrafish and pufferfish) have an inversion of the $f g f 8$ and $f b x w 4$ genes when compared to tetrapods, and are missing the btrc, poll, and dpcd genes (Fig. 2) [22]. The genome of the spotted gar, Lepisosteus oculatus, a bony fish whose lineage split before the teleost radiation, contains the same inversion of the $f g f 8$ and $f b x w 4$ genes (Fig. 2) [23]. Furthermore, the gar genome has retained the $b t r c, d p c d$ and poll genes, showing that the ancestral inversion involved a series of genes $(f g f 8, f b x w 4, d p c d$, and poll), some of which were lost in the teleost lineage (Fig. 2). This large inversion coincides with the boundaries of a topological associated domain (TAD), which are partitioned areas of the genome that define the level of interaction between genomic loci therein (Fig. 2) [24, 25]. Further insight has come from the genome sequence of the coelacanth, one of the two living groups of aquatic lobe-finned fish more closely related to tetrapods than fish [26]. The coelacanth exhibits a tetrapod-like pattern of synteny surrounding $f g f 8$, showing that the gene order present in mouse was established at least in the common ancestor of lobe-finned fishes (Fig. 2).

The common ancestor of ray-finned (actinopterygian) and lobe-finned (sarcopterygian, including tetrapods) may have had as many as 13 enhancers that drove AER activity (Fig. 2). The gar genome reveals that an inversion involving four genes occurred during the evolution of vertebrates that was not specific to teleosts, and likely involved an entire topological domain (Fig. 2). However, as the coelacanth exhibits a tetrapod-like arrangement, it is difficult to ascertain which lineage experienced the inversion event. Genome sequence from cartilaginous fishes may provide insight into how this genomic region evolved (although the synteny of the $f g f 8$ locus is elephant shark is very different in comparison to zebrafish, gar, and mouse). Regardless of when the inversion transpired, the retention of nearly all enhancers despite rearrangement shows remarkable conservation of what seem to be redundant activities (Fig. 2). The combination of functional studies of $F g f 8$ limb regulatory elements and comparisons of non-model genomic sequence reveals a complex and dynamic system of regulation for the seemingly simple structure of the AER. 


\section{Shh regulation in appendages: conservation of landscape and function}

While $F g f 8$ is crucial for outgrowth of the limb, patterning the distal skeleton is in part controlled by the genes of the Shh pathway [27]. During tetrapod limb development, Shh is expressed in a posterior patch of cells called the "Zone of Polarizing Activity" (ZPA), where it acts a morphogen to determine both digit identity and number in a concentration-dependent manner (Fig. 1)[27]. Shh is essential for the development of distal structures of the limb, as mutants show a nearly complete and specific loss of the autopod (Fig. 1)[28]. Gain of function experiments in chicks show that when $S h h$ is ectopically expressed in the anterior side of the developing forelimb, a mirror image pattern of digits develops [29].

Shh activity in the mouse limb is driven by a regulatory element lying nearly 1 megabase (MB) distant from the coding region, named the "ZPA Regulatory Element" (ZRS). The interval between $S h h$ and the ZRS contains a number of CREs that regulate Shh in other tissues such as the brain, notochord, lungs, gut and many other organs [30]. Surprisingly, the ZRS is the only known CRE that drives $S h h$ activity in the appendage. Deletion of the ZRS results in the elimination of Shh signal in the limb, making it likely that a single enhancer in the intron of a gene $1 \mathrm{MB}$ away from is responsible for $S h h$ expression in vertebrate appendages [31]. Functional studies of the ZPA have narrowed the enhancer activity to a segment of $\sim 780$ bps (which can be further broken down to "activator" and "tethering" sequences), bound in part by ETS transcription factors [32].

The ZPA has been identified in a number of fish species, including zebrafish and skate, revealing an ancient role for Shh in the patterning of vertebrate appendages [33, 34]. Both the sequence and the position of the ZRS have been highly conserved throughout vertebrate evolution (Fig. 3). In all fish that have paired appendages, the ZRS is present in the fifth intron of the gene $L m b r l$, and is separated from the Shh target gene by a large gene desert in all species ( $\sim 900 \mathrm{~kb}$ in mouse and $\sim 215 \mathrm{~kb}$ in elephant shark)(Fig. 3). As this configuration is conserved in elephant shark (a member of an ancient group of cartilaginous fish) it is likely that $S h h$ regulation by the distant action of the ZRS is a conserved feature of all jawed fishes (gnathostomes), pushing the origin of this cis regulatory program to at least $\sim 450$ mya (Fig. 3). The functional activity of the ZRS also appears conserved across vertebrates, as the ZRS from pufferfish is able to drive an identical pattern of expression in transgenic mice as the endogenous murine ZRS (Fig. 3)[35]. While this experiment suggests conservation of function, a wider taxonomic survey of ZRS elements in a transgenic context is needed to assess the level of functional conservation.

Taken together, the regulation of $S h h$ in vertebrate limbs is an extreme example of conservation on topological, cis, and trans levels (see section 5). The position of the ZRS in the fifth intron of Lmbrl is conserved in all vertebrates tested; yet the constraint acting upon its location is unclear, as the ZRS is able to act upon reporter genes that lie in different configurations across the locus [30]. Both the CREs and the TFs that bind them also appear to be conserved despite nearly 1 billion years divergence among the two lineages, as the Fugu ZRS is able to active expression identical to the native mouse 
enhancer. Do any other crucial loci in appendage development show this great level of conservation?

\section{The regulatory landscape of Hox genes in appendage evolution}

Tetrapods contain 4 clusters of Hox genes (A-D), which are responsible for patterning the primary axis in bilaterians [36]. Two of these clusters, $A$ and $D$, have crucial roles in building the secondary axis (limbs) in tetrapods [37]. The limbs of mice are built via two successive "phases" of HoxD and HoxA expression: an "early" phase that is associated with the arm and forearm, and a "late" phase that orchestrates the construction of the wrist and digits (autopod) (Fig. 1)[37]. A loss of early phase expression via gene knockout results in the absence of proximal structures, such as the forearm, while loss of the late phase results in the complete absence of the autopod (Fig. 1)[37]. The CRE's that control the early and late phases of the HoxD cluster have been elucidated and examined in detail in mouse [38-40]. Two enhancers residing 3' to the cluster control the early phase of HoxD expression, which is followed by a topological shift of chromatin allowing portions of the cluster to contact 5 ' enhancers that control late phase expression and autopod development $[38,39]$. The digit "regulatory archipelago" contains as many as seven CREs, though not all may be involved in driving late phase HoxD expression (Fig. 4) [40, 41]. HoxA activity is controlled by a similar regulatory topology, where a series of enhancers on one side of the cluster contact 5' HoxA genes to drive expression in developing digits (Fig. 4) [42, 43]. Due to their crucial role in limb development, the evolution of Hox gene expression has become a central point of study in deciphering molecular mechanisms behind the fin to limb transition $[15,44]$.

Patterns of gene expression resembling early and late phase hox activity have been described in a number of fish species, including zebrafish, paddlefish, and catshark [45-47]. Expression pattern alone cannot be taken as evidence for conservation of underlying gene regulatory mechanisms, which has shifted recent focus to the identification of early and late phase enhancers in fish genomes [44]. Expression patterns are likely homologous in two species if the genes utilize the same regulatory architecture (CREs, TFs), which are unlikely to have evolved by convergence. At the epigenomic level, the zebrafish genome contains the two topological domains at hox loci that are seen in mouse, strongly suggesting a regulatory system common in fish and tetrapods (Fig. 4)[48]. Furthermore, studies of non-model fish species have revealed the presence of mouse "digit" enhancer orthologs in the genomes of a variety of fish [41, 49]. Taken together, these studies reveal conservation at both the epigenomic and the sequence level of the regulatory network that drives Hox expression in both limbs and fins (Fig. 4).

Despite the aforementioned conservation, functional validation of fish enhancers via transgenesis have yielded conflicting results, which in turn have important implications for how we define homology and how we view the evolution of the autopod. When tested in transgenic mice, late phase enhancers from teleosts (zebrafish, Fugu, Tetraodon) drive expression only in proximal structures of the limb (or not at all), and are notably absent from the autopod (Fig. 4)[41, 48-50]. A late phase enhancer (CsB) from the little skate drives expression in the autopod of transgenic mice, but not robustly in the developing digits [49]. These observations have led to the hypothesis that although late 
phase enhancers are present in fish genomes, they are not functionally capable of activating a digit-producing program, and thus a type of "retrofitting" of fish late phase CREs was necessary to produce digits during the fin to limb transition [48]. However, CREs from the spotted gar, an actinopterygian fish that is not a teleost, are able to drive patterns of late phase expression in developing digits that are nearly identical to those of the endogenous enhancers in mouse (Fig. 4)[41]. Thus, the activities of fish enhancers, when introduced into transgenic mice, are variable. What does the diversity of patterns revealed by using different species in transgenic experiments imply for the evolution of Hox gene regulation in appendages, and for our understanding of the homology of skeletal elements in fins and limbs? The next section will discuss caveats to interpreting transgenic experiments in an evolutionary context.

\section{Inter-species transgenesis and developmental systems drift}

The proper activation of an enhancer relies on the interaction between cis (the DNA binding site), and trans (the TFs that bind it). Decades of study in the evolution of gene regulation have shown that both the sequence of the enhancer and the properties of the TFs evolve, though recent studies show greater instances of changes in cis [51, 52]. The standard and most widely used assay to determine the function of an enhancer is transgenesis, where the regulatory element of interest is placed upstream of minimal promoter and a reporter gene and injected to make a transgenic host organism. Interpreting transgenics in a native organism is more straightforward (i.e. an element from the mouse genome tested in a transgenic mouse), as the cis element is tested in its endogenous trans environment. However, evolutionary developmental biologists, who are by definition interested in a comparative approach, are faced with a more challenging interpretation of inter-species transgenesis. Biologists are limited to model systems that are amenable to the assay of interest, and reliable transgenesis is a technique that often takes years to perfect in emerging systems. The result is a nearly limitless amount of donor sequences with only a few host species with which to test them. Thus, it is often impossible to test regulatory elements in their natural host, and the necessary evil of inter-species transgenesis must be relied upon. When testing a regulatory element in any organism other than the original host, careful consideration must be taken in what can be interpreted from the results.

Transgenic enhancer experiments involve two variables: donor (the cis DNA element), and host (the organism that will receive the cis element). As mentioned previously, when donor DNA and the host organism match (i.e. mouse in mouse), reproducible patterns of activity can be attributed solely to the regulatory element tested. When the donor and host species do not match (a "swap"), interpretations must take into account potential evolution in cis, trans, or both [52]. The number and type of conclusions that can be made from a "swap" experiment have been reviewed in detail elsewhere [52]. The most relevant type of swap for this discussion is one where the activity of the donor cis element in its native trans environment is either unknown or impossible to ascertain (often the case for non-model systems). Without knowledge of the enhancer capability in its native trans environment, no negative conclusion can be made concerning the activity of that element in another host organism. Foreign CREs may fail to drive expression in a transgenic host of a different species for a number of 
reasons that are irrelevant to the evolution of phenotype. In such "non-controlled" swap experiments, evolution in trans cannot be ruled out, and thus causality or direction of evolution cannot be inferred [52]. The ability of underlying genetic systems of homologous characters to change in function is called "Developmental Systems Drift" (DSD), and is a phenomenon involving cis and trans coevolution that always must be considered when interpreting inter-species transgenic experiments [52-55].

When we consider the Hox appendage enhancer discussion in section 4 in this context, the manner in which inter-species transgenic experiments are interpreted becomes crucial for making statements about the evolutionary history of the limb. When assayed in transgenic mice, hox enhancers show a variety of different expression patterns depending on the phylogenetic position of the fish (Fig. 4 and Fig. 5)[41, 48]. While these regulatory elements have differing activities in the context of the developing mouse, all enhancers tested from fish are active in the pectoral fin of transgenic zebrafish (Fig. 5)[41, 49]. Thus, phylogenetic distance between the donor and host organism is a factor that cannot be ignored, as only when hox enhancers from some fish are placed in the context of a different trans environment (mouse) does the function drift (Fig. 5). The inability of some fish enhancers to drive expression in the autopods of transgenic mice is not due an intrinsic lack of function in these CREs, but developmental systems drift between donor and host species. Fish and tetrapods contain orthologous enhancers that may underlie homologous structures, yet the trans system of the host cannot always properly decode donor cis in a "swap" due to local evolution in each species.

\section{Conclusions}

This review has attempted to survey the evolution of regulatory control of three loci that are crucial to the development of paired appendages. This type of comparative analysis has been aided by the rise of functional genomics, allowing researchers to define entire landscapes of regulatory activity rather than a single enhancer-by-enhancer search. Comparisons of these loci and their regulatory control reveal a number of common themes.

First, and arguably most importantly, there appears to be a deep conservation of general regulatory architecture in fins and limbs. While there are degrees of flexibility in enhancer number and genomic position, in general we see the same regulatory logic in place in both fins and limbs. These commonalities are likely due to TADs, which are were likely defined early in vertebrate evolution and constraint contact between genes and their surrounding regulatory environment [56].

Second, there is a remarkably diverse level of enhancer flexibility within the general constraints of specific TADs. For example, the Fgf8 locus contains 12 enhancers that are shared between mouse and fish, a seemingly large number of regulatory elements to drive a small distal strip of gene expression in the limb and fin. Within this regulatory region, enhancers have persisted despite large local rearrangement via genomic inversion. One could argue that the plethora of $F g f 8$ enhancers allows flexibility or redundancy to buffer the activity of a gene that is required for limb development. However, the opposite situation exists in the Shh locus, a gene equally important for appendage development. Shh expression in paired appendages is regulated by a single enhancer that lies close to $1 \mathrm{MB}$ away from its promoter, in the same intron of 
the same gene in every vertebrate taxon studied. The ZRS appears "locked" into position, whereas other enhancers (such as those regulating $F g f 8$ ) appear free to diverge in relative position after inversion of the TAD. Interestingly, transposon-induced rearrangement of reporter gene position within the Shh TAD has shown that the ZPA pattern of limb expression persists despite vastly different positions of the target gene relative to the ZRS [30]. This implies that the Shh TAD does not contain strict subunits, and the TAD is a generally open domain free to receive input from individual enhancers without affecting bystander genes [30]. It remains unclear why, despite the evidence provided by these experimental manipulations, certain loci appear to be more "flexible" in their regulatory control, while others appear "locked".

Finally, while we see overall conservation of regulatory landscapes in $\mathrm{Fg} f 8, \mathrm{Sh}$, and Hox loci, it is important to note the potential effects of enhancer number and/or activity potential within these domains. The HoxD and HoxA clusters of mouse appear to have a number of tetrapod-specific enhancers in the "digit archipelago", implying that gain of additional late phase enhancers (such as $\mathrm{CsC}$ ) may have had a role in the fin to limb transition and the evolution of the autopod (Fig. 4)[57]. However, as the presence/absence of these enhancers is based only on sequence conservation, an exhaustive definition of the complete regulatory landscape of hox genes in multiple fish species is necessary to ascertain whether there was a gain in enhancer number throughout evolution of the autopod. In addition, evolutionarily conserved enhancers have been found to evolve new intrinsic functions, again making it clear that functional assays must be performed when defining enhancers [58]. As functional assays are critical to elucidating enhancer landscapes, and often take the form of transgenesis between species, we have argued here for the careful analysis of the results of such experiments. DSD may make enhancers between species incompatible when swapped, even through they pattern homologous structures with conserved regulatory inputs in their native environments. Transgenesis in the native organism (or one as phylogenetically close as possible) must always be performed to make a controlled swap [52].

\section{Future directions: a phylogenetic perspective on functional genomics}

The development of high-throughput, whole-genome technologies that require a relatively small (or even single) input of cells has a potentially transformative impact on evolutionary studies [59-63]. Developmental biologists interested in the evolution of phenotype have the potential to complement the work done in model species, such as chicks and mice, with work on an ever-increasing diversity of species that was previously impossible due to technical limitations of bulk tissue needed. Armed with these tools, virtually any species has the potential to become a "model" of sorts. By being able to select taxa with relevant phenotypes, phylogenetic histories, and genomic structures, biologists can bring increasing granularity to comparisons, thereby allowing ever more refined tests of hypotheses of the genetic basis of morphological transformations. Finally, a greater understanding of regulatory landscapes across taxa will identify meaningful targets for direct genomic manipulation in order to test hypotheses of evolutionary scenarios [64]. Functional genomics, performed in the context of as wide a phylogenetic breadth as possible, will provide valuable insight for the future of evo devo. 


\section{Acknowledgements}

We are grateful for artwork and aid in figure assembly by John Westlund. We thank Kacy Gordon and Robert Arthur for critical reading of the manuscript. A.R.G is supported by National Institutes of Health Grant T32 HD055164 and National Science Foundation Doctoral Dissertation Improvement Grant 1311436. N.H.S would like to acknowledge funding from the Brinson Foundation and the University of Chicago Biological Sciences Division. 


\section{References}

[1] Wray GA, Hahn MW, Abouheif E, Balhoff JP, Pizer M, Rockman MV, et al. The evolution of transcriptional regulation in eukaryotes. Mol Biol Evol 2003;20:1377-419. [2] Stewart AJ, Hannenhalli S, Plotkin JB. Why transcription factor binding sites are ten nucleotides long. Genetics 2012;192:973-85.

[3] Bulyk ML. Computational prediction of transcription-factor binding site locations. Genome biology 2003;5:201.

[4] Carroll SB. Evo-devo and an expanding evolutionary synthesis: a genetic theory of morphological evolution. Cell 2008;134:25-36.

[5] Wray GA. The evolutionary significance of cis-regulatory mutations. Nat Rev Genet 2007;8:206-16.

[6] Chan YF, Marks ME, Jones FC, Villarreal G, Jr., Shapiro MD, Brady SD, et al. Adaptive evolution of pelvic reduction in sticklebacks by recurrent deletion of a Pitx1 enhancer. Science 2010;327:302-5.

[7] Stern DL, Frankel N. The structure and evolution of cis-regulatory regions: the shavenbaby story. Philosophical transactions of the Royal Society of London Series B, Biological sciences 2013;368:20130028.

[8] de Wit E, de Laat W. A decade of 3C technologies: insights into nuclear organization. Genes Dev 2012;26:11-24.

[9] Park PJ. ChIP-seq: advantages and challenges of a maturing technology. Nat Rev Genet 2009;10:669-80.

[10] Buenrostro JD, Giresi PG, Zaba LC, Chang HY, Greenleaf WJ. Transposition of native chromatin for fast and sensitive epigenomic profiling of open chromatin, DNAbinding proteins and nucleosome position. Nat Methods 2013;10:1213-8.

[11] Pombo A, Dillon N. Three-dimensional genome architecture: players and mechanisms. Nat Rev Mol Cell Biol 2015;16:245-57.

[12] Niswander L, Tickle C, Vogel A, Booth I, Martin GR. FGF-4 replaces the apical ectodermal ridge and directs outgrowth and patterning of the limb. Cell 1993;75:579-87. [13] Fallon JF, Lopez A, Ros MA, Savage MP, Olwin BB, Simandl BK. FGF-2: apical ectodermal ridge growth signal for chick limb development. Science 1994;264:104-7. [14] Mariani FV, Ahn CP, Martin GR. Genetic evidence that FGFs have an instructive role in limb proximal-distal patterning. Nature 2008;453:401-U56.

[15] Schneider I, Shubin NH. The origin of the tetrapod limb: from expeditions to enhancers. Trends Genet 2013;29:419-26.

[16] Thorogood P. The development of the teleost fin and implications for our understanding of tetrapod limb evolution. Developmental Patterning of the Vertebrate Limb: Plenum Press; 1991. p. 347-54.

[17] Freitas R, Zhang G, Cohn MJ. Evidence that mechanisms of fin development evolved in the midline of early vertebrates. Nature 2006;442:1033-7.

[18] Grandel H, Draper BW, Schulte-Merker S. dackel acts in the ectoderm of the zebrafish pectoral fin bud to maintain AER signaling. Development 2000;127:4169-78. 
[19] Grandel H, Schulte-Merker S. The development of the paired fins in the zebrafish (Danio rerio). Mechanisms of development 1998;79:99-120.

[20] Marinic M, Aktas T, Ruf S, Spitz F. An integrated holo-enhancer unit defines tissue and gene specificity of the fgf8 regulatory landscape. Dev Cell 2013;24:530-42.

[21] Beermann F, Kaloulis K, Hofmann D, Murisier F, Bucher P, Trumpp A.

Identification of evolutionarily conserved regulatory elements in the mouse Fgf8 locus.

Genesis 2006;44:1-6.

[22] Komisarczuk AZ, Kawakami K, Becker TS. Cis-regulation and chromosomal rearrangement of the fgf8 locus after the teleost/tetrapod split. Dev Biol 2009;336:30112.

[23] Braasch I, Peterson SM, Desvignes T, McCluskey BM, Batzel P, Postlethwait JH. A new model army: Emerging fish models to study the genomics of vertebrate Evo-Devo. Journal of experimental zoology Part B, Molecular and developmental evolution 2015;324:316-41.

[24] Nora EP, Lajoie BR, Schulz EG, Giorgetti L, Okamoto I, Servant N, et al. Spatial partitioning of the regulatory landscape of the X-inactivation centre. Nature 2012;485:381-5.

[25] Dixon JR, Selvaraj S, Yue F, Kim A, Li Y, Shen Y, et al. Topological domains in mammalian genomes identified by analysis of chromatin interactions. Nature 2012;485:376-80.

[26] Amemiya CT, Alfoldi J, Lee AP, Fan S, Philippe H, Maccallum I, et al. The African coelacanth genome provides insights into tetrapod evolution. Nature 2013;496:311-6.

[27] Zeller R, Lopez-Rios J, Zuniga A. Vertebrate limb bud development: moving towards integrative analysis of organogenesis. Nat Rev Genet 2009;10:845-58.

[28] Litingtung Y, Dahn RD, Li Y, Fallon JF, Chiang C. Shh and Gli3 are dispensable for limb skeleton formation but regulate digit number and identity. Nature 2002;418:979-83. [29] Riddle RD, Johnson RL, Laufer E, Tabin C. Sonic hedgehog mediates the polarizing activity of the ZPA. Cell 1993;75:1401-16.

[30] Anderson E, Devenney PS, Hill RE, Lettice LA. Mapping the Shh long-range regulatory domain. Development 2014;141:3934-43.

[31] Sagai T, Hosoya M, Mizushina Y, Tamura M, Shiroishi T. Elimination of a longrange cis-regulatory module causes complete loss of limb-specific Shh expression and truncation of the mouse limb. Development 2005;132:797-803.

[32] Lettice LA, Williamson I, Devenney PS, Kilanowski F, Dorin J, Hill RE.

Development of five digits is controlled by a bipartite long-range cis-regulator.

Development 2014;141:1715-25.

[33] Dahn RD, Davis MC, Pappano WN, Shubin NH. Sonic hedgehog function in chondrichthyan fins and the evolution of appendage patterning. Nature 2007;445:311-4. [34] Krauss S, Concordet JP, Ingham PW. A functionally conserved homolog of the Drosophila segment polarity gene hh is expressed in tissues with polarizing activity in zebrafish embryos. Cell 1993;75:1431-44.

[35] Lettice LA, Heaney SJ, Purdie LA, Li L, de Beer P, Oostra BA, et al. A long-range Shh enhancer regulates expression in the developing limb and fin and is associated with preaxial polydactyly. Human molecular genetics 2003;12:1725-35.

[36] Duboule D. The rise and fall of Hox gene clusters. Development 2007;134:2549-60. 
[37] Zakany J, Duboule D. The role of Hox genes during vertebrate limb development. Curr Opin Genet Dev 2007;17:359-66.

[38] Lonfat N, Duboule D. Structure, function and evolution of topologically associating domains (TADs) at HOX loci. FEBS Lett 2015.

[39] Andrey G, Montavon T, Mascrez B, Gonzalez F, Noordermeer D, Leleu M, et al. A switch between topological domains underlies HoxD genes collinearity in mouse limbs.

Science 2013;340:1234167.

[40] Montavon T, Soshnikova N, Mascrez B, Joye E, Thevenet L, Splinter E, et al. A regulatory archipelago controls Hox genes transcription in digits. Cell 2011;147:1132-45. [41] Gehrke AR, Schneider I, de la Calle-Mustienes E, Tena JJ, Gomez-Marin C, Chandran M, et al. Deep conservation of wrist and digit enhancers in fish. Proc Natl Acad Sci U S A 2015;112:803-8.

[42] Berlivet S, Paquette D, Dumouchel A, Langlais D, Dostie J, Kmita M. Clustering of tissue-specific sub-TADs accompanies the regulation of HoxA genes in developing limbs. Plos Genet 2013;9:e1004018.

[43] Lehoczky JA, Innis JW. BAC transgenic analysis reveals enhancers sufficient for Hoxa13 and neighborhood gene expression in mouse embryonic distal limbs and genital bud. Evol Dev 2008;10:421-32.

[44] Woltering JM, Duboule D. The Origin of Digits: Expression Patterns versus Regulatory Mechanisms. Dev Cell 2010;18:526-32.

[45] Ahn D, Ho RK. Tri-phasic expression of posterior Hox genes during development of pectoral fins in zebrafish: implications for the evolution of vertebrate paired appendages. Dev Biol 2008;322:220-33.

[46] Davis MC, Dahn RD, Shubin NH. An autopodial-like pattern of Hox expression in the fins of a basal actinopterygian fish. Nature 2007;447:473-6.

[47] Freitas R, Zhang G, Cohn MJ. Biphasic Hoxd gene expression in shark paired fins reveals an ancient origin of the distal limb domain. PLoS One 2007;2:e754.

[48] Woltering JM, Noordermeer D, Leleu M, Duboule D. Conservation and divergence of regulatory strategies at hox Loci and the origin of tetrapod digits. PLoS Biol 2014;12:e1001773.

[49] Schneider I, Aneas I, Gehrke AR, Dahn RD, Nobrega MA, Shubin NH. Appendage expression driven by the Hoxd Global Control Region is an ancient gnathostome feature. Proc Natl Acad Sci U S A 2011;108:12782-6.

[50] Spitz F, Gonzalez F, Duboule D. A global control region defines a chromosomal regulatory landscape containing the HoxD cluster. Cell 2003;113:405-17.

[51] Wittkopp PJ, Haerum BK, Clark AG. Evolutionary changes in cis and trans gene regulation. Nature 2004;430:85-8.

[52] Gordon KL, Ruvinsky I. Tempo and mode in evolution of transcriptional regulation. Plos Genet 2012;8:e1002432.

[53] True JR, Haag ES. Developmental system drift and flexibility in evolutionary trajectories. Evol Dev 2001;3:109-19.

[54] Verster AJ, Ramani AK, McKay SJ, Fraser AG. Comparative RNAi screens in C. elegans and C. briggsae reveal the impact of developmental system drift on gene function. Plos Genet 2014;10:e1004077. 
[55] Wang X, Sommer RJ. Antagonism of LIN-17/Frizzled and LIN-18/Ryk in nematode vulva induction reveals evolutionary alterations in core developmental pathways. PLoS Biol 2011;9:e1001110.

[56] Dekker J, Heard E. Structural and functional diversity of Topologically Associating Domains. FEBS Lett 2015.

[57] Freitas R, Gomez-Marin C, Wilson JM, Casares F, Gomez-Skarmeta JL. Hoxd13 contribution to the evolution of vertebrate appendages. Dev Cell 2012;23:1219-29.

[58] Koshikawa S, Giorgianni MW, Vaccaro K, Kassner VA, Yoder JH, Werner T, et al. Gain of cis-regulatory activities underlies novel domains of wingless gene expression in Drosophila. Proc Natl Acad Sci U S A 2015;112:7524-9.

[59] Schmidl C, Rendeiro AF, Sheffield NC, Bock C. ChIPmentation: fast, robust, lowinput ChIP-seq for histones and transcription factors. Nat Methods 2015.

[60] Cao Z, Chen C, He B, Tan K, Lu C. A microfluidic device for epigenomic profiling using 100 cells. Nat Methods 2015.

[61] Buenrostro JD, Wu B, Litzenburger UM, Ruff D, Gonzales ML, Snyder MP, et al. Single-cell chromatin accessibility reveals principles of regulatory variation. Nature 2015;523:486-90.

[62] Cusanovich DA, Daza R, Adey A, Pliner HA, Christiansen L, Gunderson KL, et al. Epigenetics. Multiplex single-cell profiling of chromatin accessibility by combinatorial cellular indexing. Science 2015;348:910-4.

[63] Brind'Amour J, Liu S, Hudson M, Chen C, Karimi MM, Lorincz MC. An ultra-lowinput native ChIP-seq protocol for genome-wide profiling of rare cell populations. Nat Commun 2015;6:6033.

[64] Pieretti J, Gehrke AR, Schneider I, Adachi N, Nakamura T, Shubin NH. Organogenesis in deep time: A problem in genomics, development, and paleontology. Proc Natl Acad Sci U S A 2015;112:4871-6.

[65] Moon AM, Capecchi MR. Fgf8 is required for outgrowth and patterning of the limbs. Nat Genet 2000;26:455-9.

[66] Lewandoski M, Sun X, Martin GR. Fgf8 signalling from the AER is essential for normal limb development. Nat Genet 2000;26:460-3.

[67] Summerbell D, Lewis JH, Wolpert L. Positional information in chick limb morphogenesis. Nature 1973;244:492-6.

[68] Guo Y, Xu Q, Canzio D, Shou J, Li J, Gorkin DU, et al. CRISPR Inversion of CTCF Sites Alters Genome Topology and Enhancer/Promoter Function. Cell 2015;162:900-10. [69] Frazer KA, Pachter L, Poliakov A, Rubin EM, Dubchak I. VISTA: computational tools for comparative genomics. Nucleic Acids Res 2004;32:W273-9. 


\section{Figure legends}

Fig. 1. Summary of vertebrate appendage development and mouse mutants highlighting the role of key genes. A) The forelimbs of mice and the pectoral fins of zebrafish utilize similar gene networks during development; $F g f s$ in the AER, Shh expression in the posterior mesenchyme, and both early and late phases of Hox gene expression. Late phase Hox expression (both hoxa and hoxd) is shown based on the activity pattern of late phase enhancers in zebrafish described in [41]. The blue and red colors are meant to denote the area in which early and late phases are active, and not the specific nested patterns of individual Hox genes. Note the early fin fold in the 48 hpf zebrafish fin (shown in yellow to denote $f g f 8 a$ expression), a structure that does not develop in tetrapods. B) Despite these conserved expression patterns, tetrapod limbs and zebrafish fins are morphologically disparate. The mouse limb is composed entirely of endochondral bone, while the zebrafish fin consists of a base of endochondrol bone followed by a long series of fin rays consisting of dermal bone. C) Mutant mice underscore the key nature of the genes discussed. Conditional knockouts of $F g f 8$ in mouse results in either a lack of the stylopod and parts of the zeugopod [65], or are smaller and missing distal structures [66]. Removal of the AER (and thus Fgf activity) in chicken results in the arrest of limb development, in a time dependent context [67]. Mice lacking Shh expression the limb fail to develop an autopod, leaving only a single ambiguous digit [28]. Loss of early phase HoxD and HoxA expression leads to a loss of proximal structures of the limb, while loss of late phase expression results in complete deletion of the autopod [37].

Fig. 2. The evolution of the $F g f 8$ regulatory landscape. Blue boxes denote enhancers that were identified and tested in zebrafish, red boxes indicate those that were identified and tested in mouse. Multicolored boxes indicate the same enhancers that were found independently in mouse and fish. In some instances, the murine ortholog of a validated 
zebrafish enhancer did not drive expression in mouse (e.g. CE62 from mouse). The enhancer that did not show activity in transgenic mice is denoted by a lighter color. An inversion of the Poll, Dpcd, Fbxw4, and Fgf8 genes took place either in the ancestor of bony fish (Actinopterygii) or in the ancestor of lobe-finned fish (Sarcopterygii) (denoted by grey segment). This was likely possible due to the inversion of an entire topological domain encompassing these genes, which can be seen in mouse Hi-C data (top). This topological domain is flanked by CTCF sites, a protein with a known role in chromatin organization that often marks the boundaries of topological domains [68]. Mouse genes are drawn according to scale to match the mouse $\mathrm{Hi}-\mathrm{C}$ data, while the size of fish genes are stylized in order to best emphasize enhancer location and gene loss. Orthologs of previously reported enhancers were identified using the mVista LAGAN program, calc window: 100 bps; min cons width: 100 bps; cons identity: 70\% [69]. Mouse Hi-C data and CTCF ChIP-seq images are from the 3D genome browser (http://www.3dgenome.org) according to mm9 region chr19:45,430,000-45,830,000.

Fig. 3. Shh expression in the paired appendages of vertebrates is controlled by a single regulatory element with deeply conserved topology and function. A) The Shh regulatory landscape showing the position of the ZRS in the fifth intron of Lmbrl. This enhancer traverses a topological domain (denoted by red triangle according to [30]) of nearly $1 \mathrm{MB}$ in mouse to contact its target gene, a long distance regulation that is conserved in all vertebrates with paired appendages. B) The ZRS is functionally conserved, as the ZRS from $F u g u$ is able to drive an identical pattern of expression to the endogenous enhancer in mouse [35].

Fig. 4. The evolutionary history of Hox gene regulation in distal appendages. A) Digit expression of Hoxd13 and Hoxal3 in mouse is driven by a series of enhancers that lie specifically on one side of the Hox cluter, spread over a distance of $\sim 500 \mathrm{~kb}$. These regulatory elements fall with topological associated domains (shown as red triangles) that define the regulatory landscape of Hox expression [40, 42]. Studies in zebrafish have shown that the same topological domains are present in fish [41, 48], along with orthologs of specific mouse digit enhancers [41, 49]. Potential mouse-specific enhancers are shown in blue, those that are shared with fish are colored purple. For the zebrafish/gar HoxA cluster, the topological domain was found in zebrafish, while the orthologs of the enhancers represented are from the gar genome. B) Summary of transgenic experiments with Hox digit enhancers. Cis-elements from mouse are denoted in blue, those from zebrafish.

Fig. 5. Interpreting inter-species transgenics in a phylogenetic context. A potential evolutionary scenario for the cis/trans interaction of the Island I Hox enhancer based on reciprocal transgenics [41]. Here, zebrafish likely underwent evolution in both cis and trans (developmental systems drift), making the host trans environment of mouse incapable of interpreting the cis element of zebrafish. 

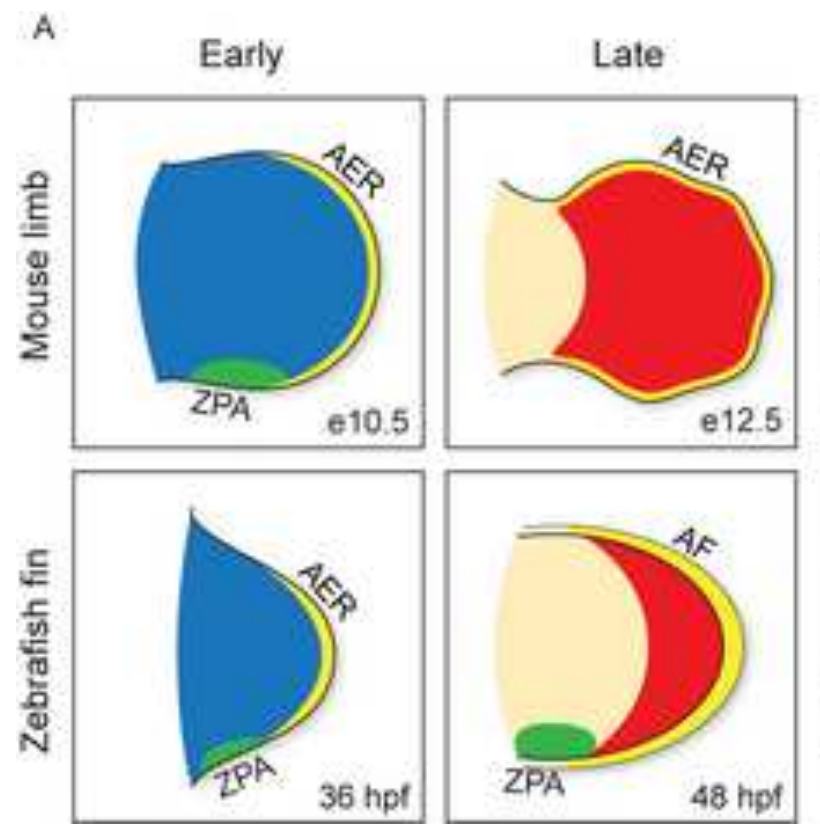

B Wildtype forelimbs
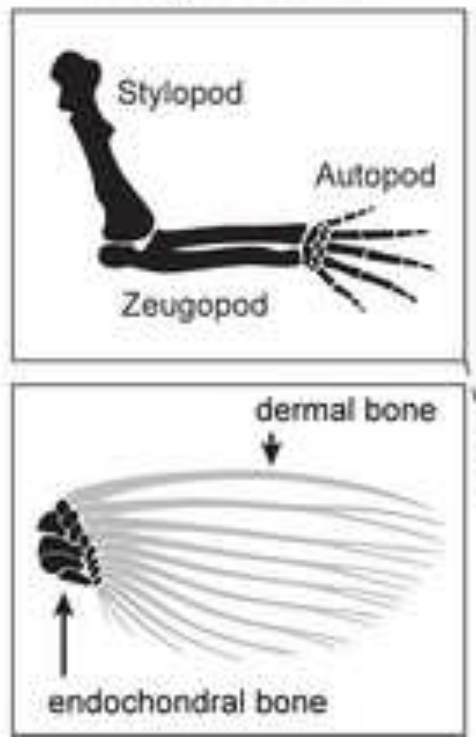

Mutant forelimbs
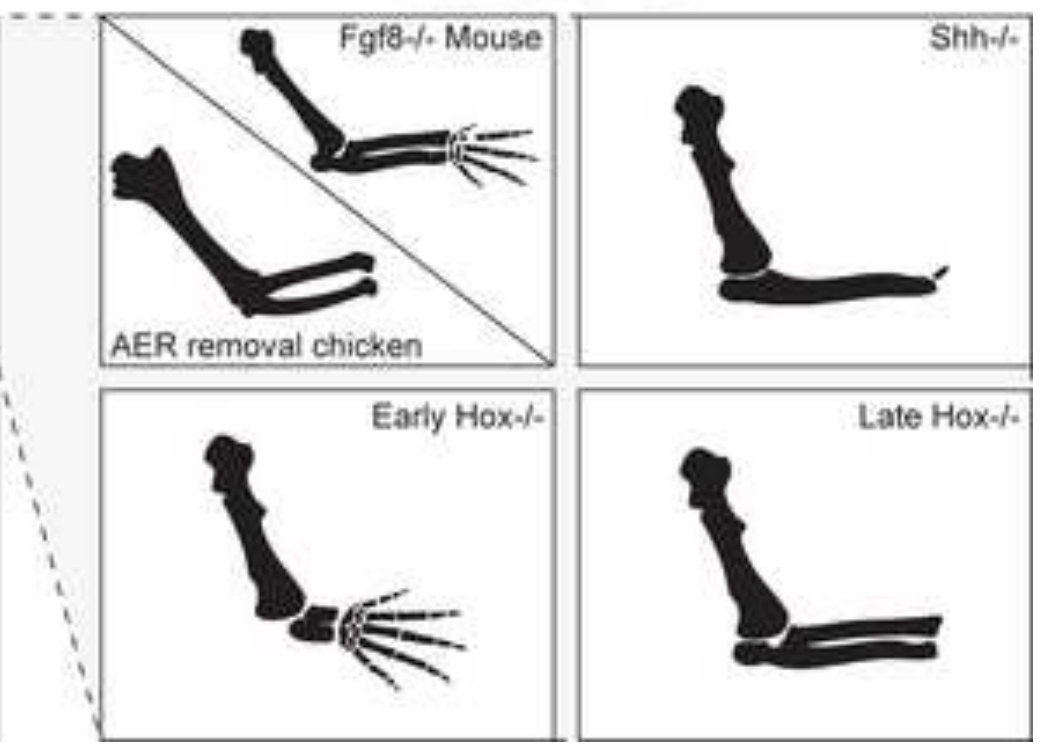


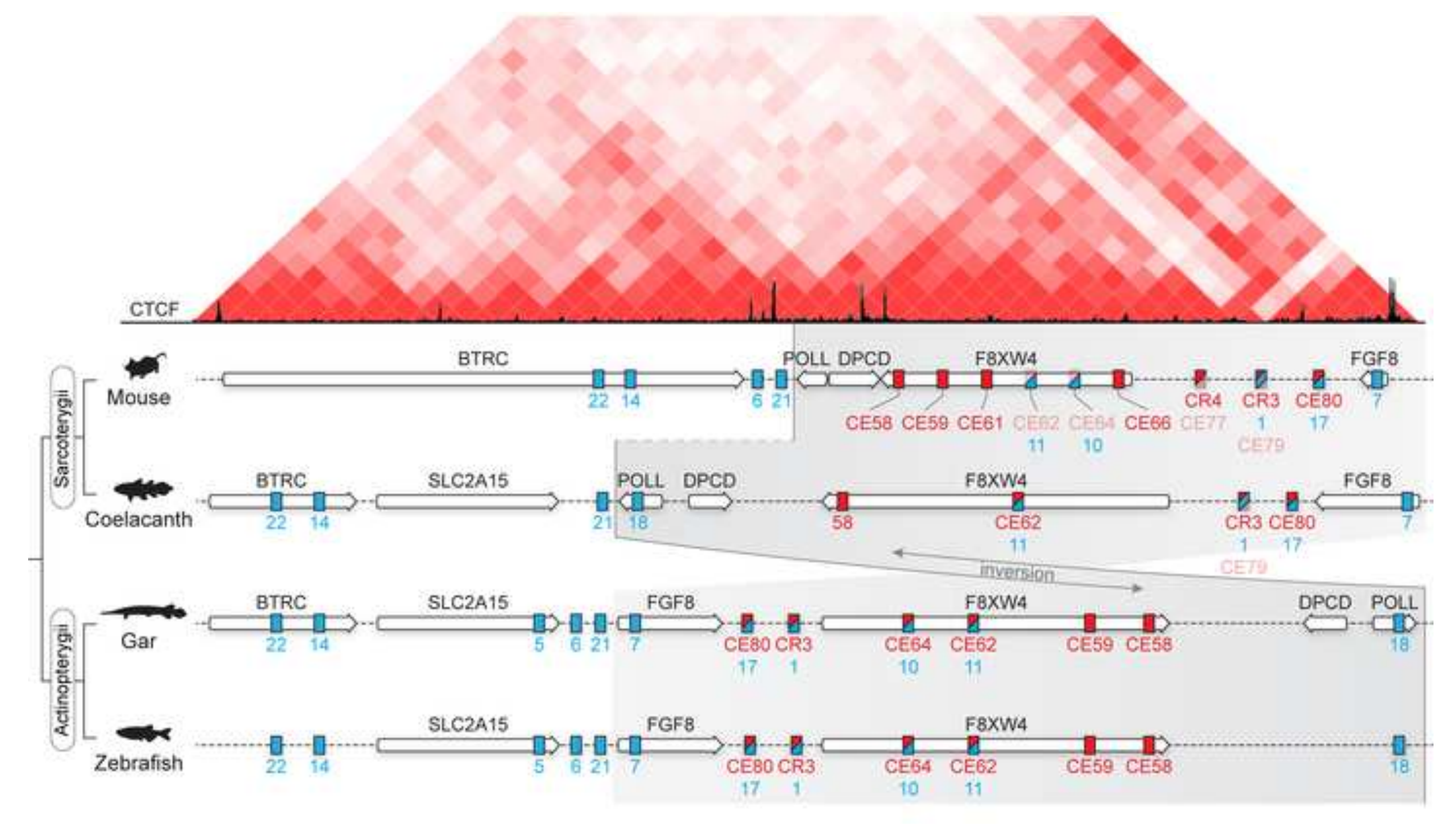

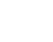



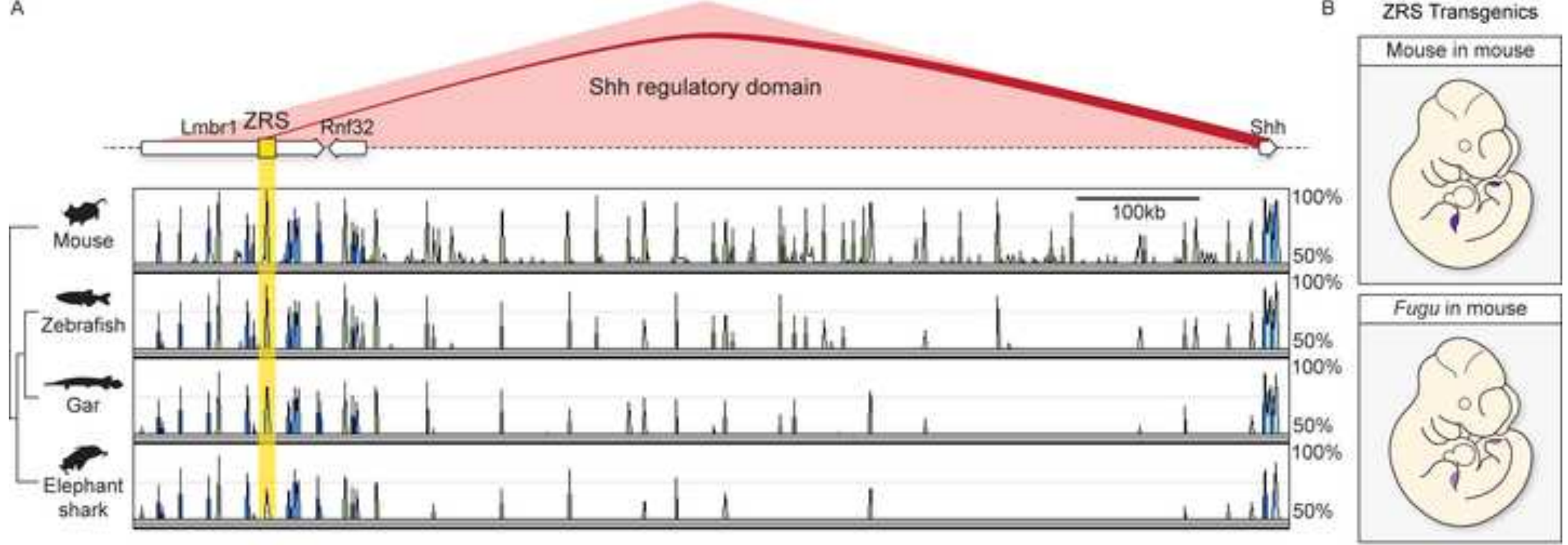
A

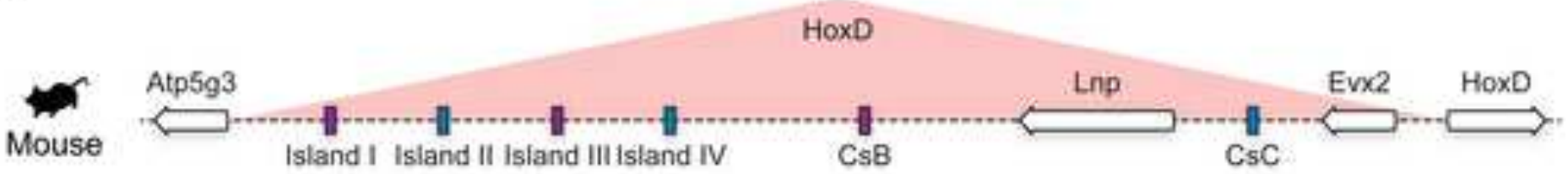

HoxD

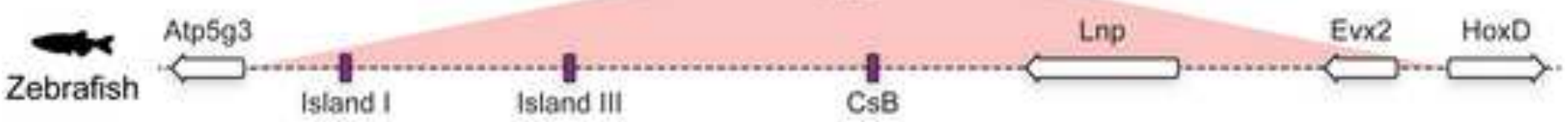

HoxA

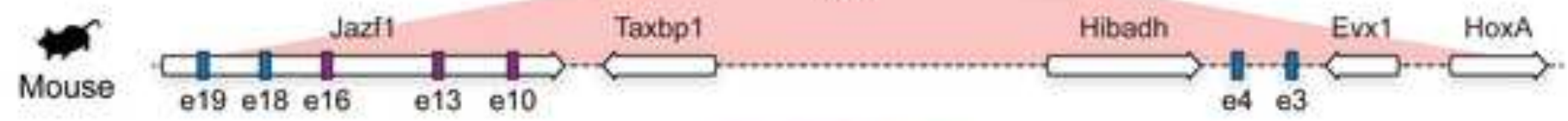

HoxA

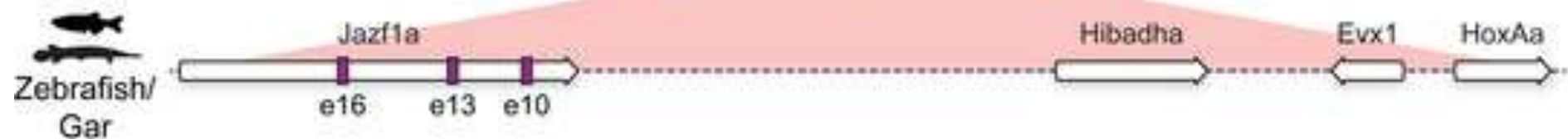

B
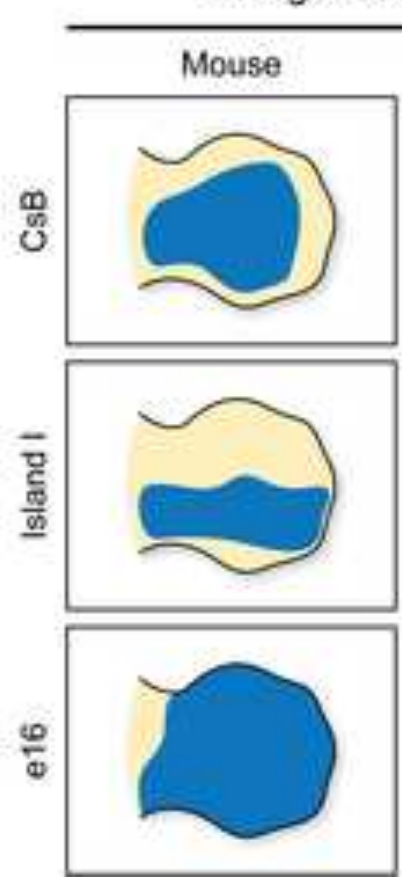

Inter-species Transgenesis

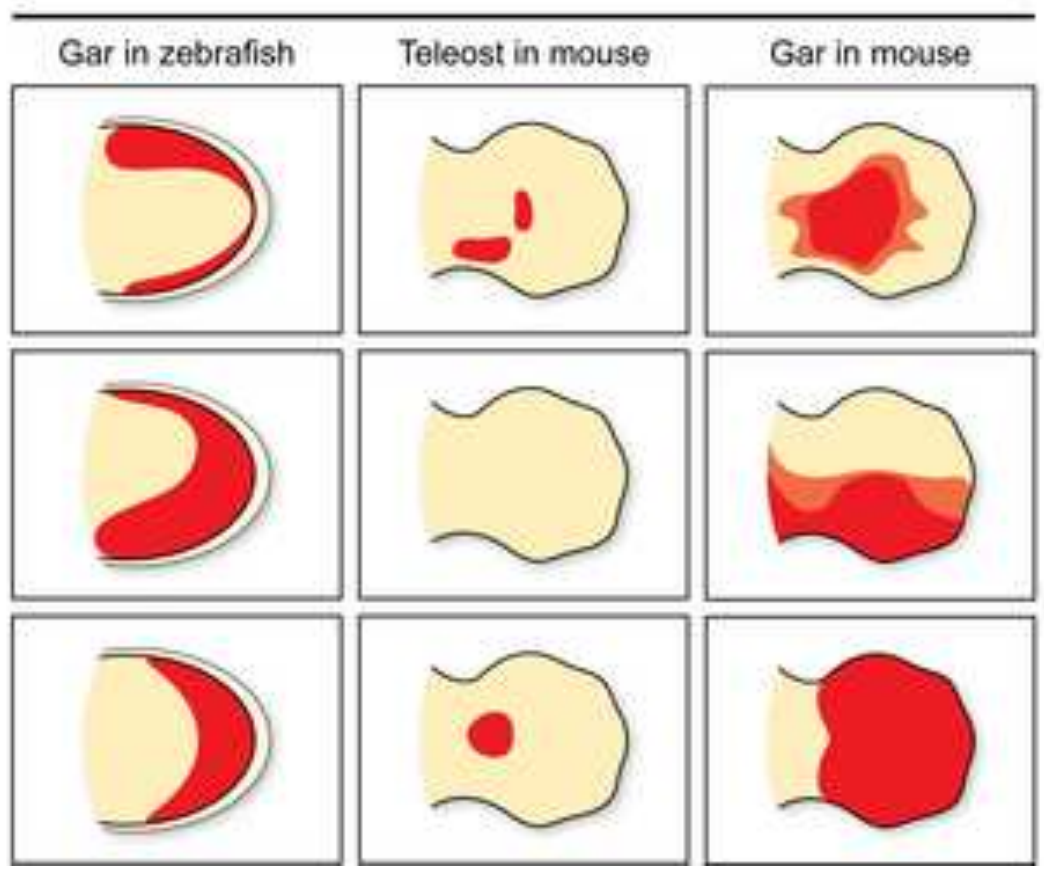




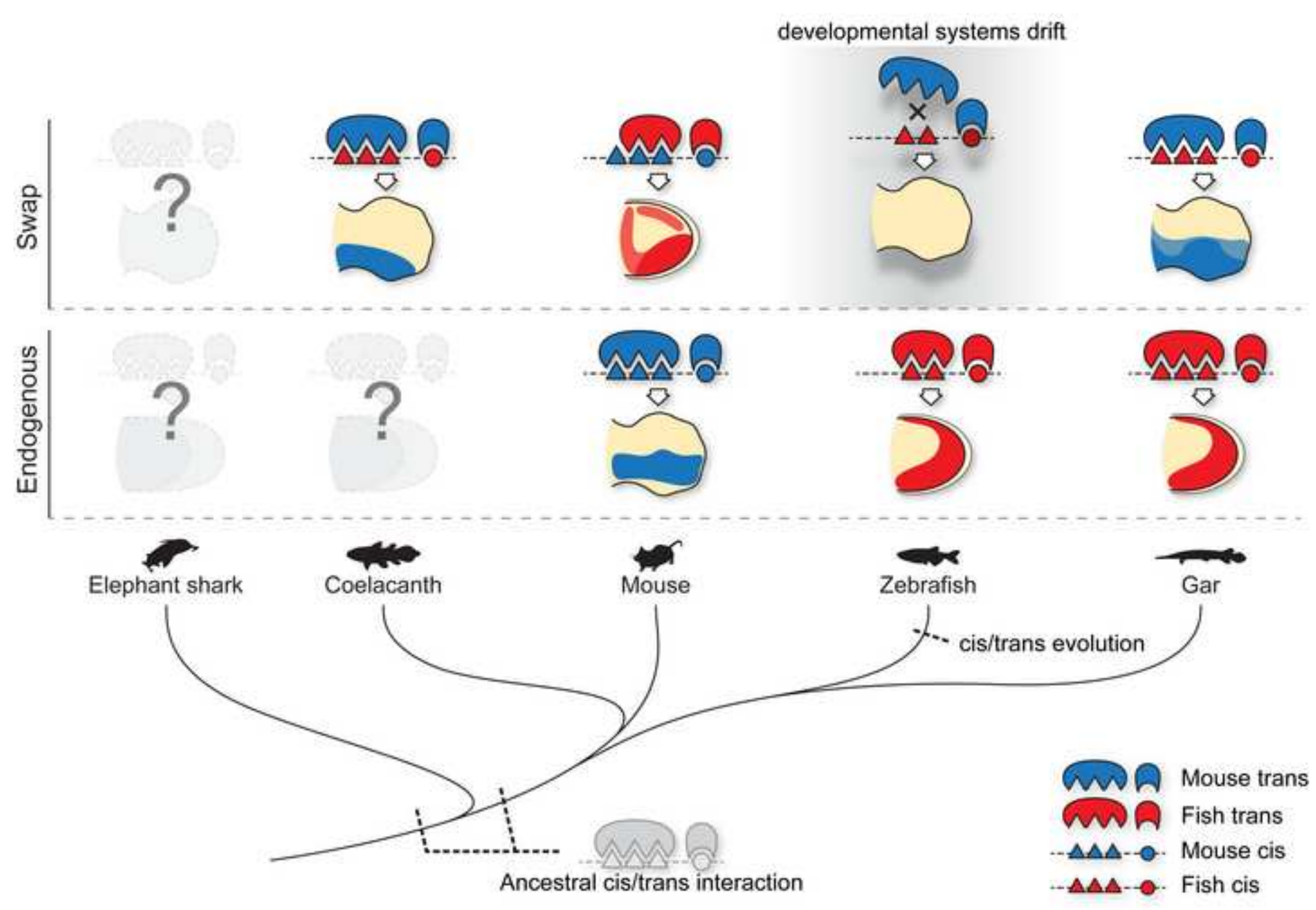

